\title{
Ursodeoxycholic Acid Induces Death Receptor-mediated Apoptosis in Prostate Cancer Cells
}

\author{
Won Sup Lee ${ }^{1,4, *}$, Ji Hyun Jung ${ }^{1,4}$, Radha Panchanathan ${ }^{1,4}$, Jeong Won Yun ${ }^{1,4}$, Dong Hoon Kim ${ }^{2,4}$, Hye Jung Kim ${ }^{3,4}$, \\ Gon Sup Kim ${ }^{5,6}$, Chung Ho Ryu ${ }^{7}$, Sung Chul Shin ${ }^{6,8}$, Soon Chan Hong ${ }^{4,9}$, Yung Hyun Choi ${ }^{10,11}$, Jin-Myung Jung ${ }^{4,12, *}$ \\ Departments of ${ }^{1}$ Internal Medicine, ${ }^{2}$ Emergency Medicine, and ${ }^{3}$ Pharmacology, Gyeongsang National University School of Medicine, ${ }^{4}$ Institute \\ of Health Sciences, Gyeongsang National University, ${ }^{5}$ School of Veterinary Medicine, ${ }^{6}$ Research Institute of Life Science, Gyeongsang National \\ University, ${ }^{7}$ Division of Applied Life Science (BK 21 Program), Institute of Agriculture and Life Science, Gyeongsang National University, ${ }^{8}$ Department \\ of Chemistry, College of Natural Sciences, Gyeongsang National University, ${ }^{9}$ Department of Surgery, Gyeongsang National University School of \\ Medicine, Jinju, ${ }^{10}$ Department of Biochemistry, Dongeui University College of Oriental Medicine, ${ }^{11}$ Anti-Aging Research Center \& Blue-Bio Industry \\ RIC, Dongeui University, Busan, ${ }^{12}$ Department of Neurosurgery, Gyeongsang National University School of Medicine, Jinju, Korea
}

Background: Bile acids have anti-cancer properties in a certain types of cancers. We determined anticancer activity and its underlying molecular mechanism of ursodeoxycholic acid (UDCA) in human DU145 prostate cancer cells.

Methods: Cell viability was measured with an MTT assay. UDCA-induced apoptosis was determined with flow cytometric analysis. The expression levels of apoptosis-related signaling proteins were examined with Western blotting.

Results: UDCA treatment significantly inhibited cell growth of DU145 in a dose-dependent manner. It induced cellular shrinkage and cytoplasmic blebs and accumulated the cells with sub-G1 DNA contents. Moreover, UDCA activated caspase 8, suggesting that UDCA-induced apoptosis is associated with extrinsic pathway. Consistent to this finding, UDCA increased the expressions of tumor necrosis factor-related apoptosis-inducing ligand (TRAIL) receptor, death receptor 4 (DR4) and death receptor 5 (DR5), and TRAIL augmented the UDCA-induced cell death in DU145 cells. In addition, UDCA also increased the expressions of Bax and cytochrome $c$ and decreased the expression of BCl-xL in DU145 cells. This finding suggests that UDCA-induced apoptosis may be involved in intrinsic pathway. Conclusions: UDCA induces apoptosis via extrinsic pathway as well as intrinsic pathway in DU145 prostate cancer cells. UDCA may be a promising anti-cancer agent against prostate cancer.

(J Cancer Prev 2017;22:16-21)

Key Words: Ursodeoxycholic acid, DU145, Apoptosis, Tumor necrosis factor-related apoptosis-inducing ligand, Prostate cancer

\section{INTRODUCTION}

Prostate cancer is one of the most prevalent cancers in men worldwide. High fat and red meat diet is one of the contributing factors to the development of prostate cancer. Notably, it is the second leading cause of cancer death for men in most developed countries. ${ }^{1}$ Also, it is the second most common cancer in men, about an estimated 192,000 and 346,000 new cases diagnosed each year in the United States and Europe, respectively. In addition, most of cases are elderly patients. These elderly patients cannot tolerate conventional chemotherapy. The prostate cancer refractory to hormonal therapy is hard to treat.

Received December 28, 2016, Revised February 17, 2017, Accepted February 17, 2017

Correspondence to: Won Sup Lee

Department of Internal Medicine, Institute of Health Sciences and Gyeongnam Regional Cancer Center, Gyeongsang National University School of Medicine,

15 Jinju-daero 816beon-gil, Jinju 52727, Korea

Tel: +82-55-750-8733, Fax: +82-55-758-9122, E-mail: lwshmo@gshp.gsnu.ac.kr

Correspondence to: Jin-Myung Jung

Department of Neurosurgery, Gyeongsang National University School of Medicine, 15 Jinju-daero 816beon-gil, Jinju 52727, Korea

Tel: +82-55-750-8110, Fax: +82-55-758-9122, E-mail: gnuhjjm@gnu.ac.kr

*These authors contributed equally to this paper as co-correspondence authors

Copyright (c) 2017 Korean Society of Cancer Prevention

(c) This is an Open Access article distributed under the terms of the Creative Commons Attribution Non-Commercial License (http://creativecommons.org/licenses/by-nc/4.0) which permits unrestricted non-commercial use, distribution, and reproduction in any medium, provided the original work is properly cited. 
Therefore, new therapeutic strategy is required for the treatment of this disease.

Bile acids is the outcome of cholesterol catabolism, and their main function is to solubilize the dietary fats and fat-soluble vitamins from the intestinal lumen. ${ }^{2,3}$ Reports have shown that bile acids possess anti-neoplastic and anti-carcinogenic properties in many cancer cells, such as tamoxifen-resistant breast cancer, colon cancer, prostate cancer, and neuroblastoma cells. ${ }^{4}$ Among them, ursodeoxycholic acid (UDCA) is a hydrophobic bile acid that accounts for $4 \%$ of total bile acids, ${ }^{5}$ and has been used for treatment of the liver and gall bladder disease without a significant toxicity. The tumor suppressive activity of UDCA is believed to be linked with the induction of apoptosis and cell cycle arrest, and the inhibition of oncogenic factors, such as Ras and COX-2, in human cancer cell lines. ${ }^{6}$ These mechanisms are not yet fully understood. In addition, evidence suggests that anti-cancer effect of UDCA may act differentially depending on cell types, physiologic conditions, and/or stimulus. ${ }^{5.7}$ Here, we investigated anti-cancer effect of UDCA and its mechanisms on human prostate cancer cells, DU145. The results show that UDCA increased the expressions of tumor necrosis factor-related apoptosis-inducing ligand (TRAIL) receptors, death receptor 4 (DR4) and death receptor 5 (DR5), and TRAIL augmented the UDCA-induced cell death in DU145 cells. To our knowledge, this is the first study reporting UDCA-induced death receptor-mediated apoptosis in human prostate cancer cells.

\section{MATERIALS AND METHODS}

\section{Cells and reagents}

Human DU145 prostate cancer cells were obtained from the American Type Culture Collection (Rockville, MD, USA). Culture medium used throughout these experiments was RPMI 1640 medium (Invitrogen, Carlsbad, CA, USA) supplemented with 10\% (v/v) FBS (GIBCO BRL, Grand Island, NY, USA), 1 mM L-glutamine, $100 \mathrm{U} / \mathrm{mL}$ penicillin, and $100 \mu \mathrm{g} / \mathrm{mL}$ streptomycin at $37^{\circ} \mathrm{C}$ in a humidified atmosphere of $95 \%$ air and $5 \% \mathrm{CO}_{2}$. UDCA was obtained from Dawoong Inc. (Seoul, Korea), and kept at $4^{\circ} \mathrm{C}$ with light shield. Antibodies against procaspase 3, procaspase 8, Bax, Bcl-xL, cytochrome $c$, PARP, and TRAIL receptors (DR4 and DR5) were purchased from Santa Cruz Biotechnology (Santa Cruz, CA, USA). Peroxidase-labeled donkey anti-rabbit and sheep anti-mouse immunoglobulins and an enhanced chemiluminescence (ECL) kit were purchased from Amersham (Arlington Heights, IL, USA). All other chemicals not specifically cited here were purchased from Sigma Chemical Co. (St. Louis, MO, USA). Stock solutions of 4',6-diamidino-2-phenylindole $(100 \mu \mathrm{g} / \mathrm{mL})$ and propidium iodide (PI, $1 \mathrm{mg} / \mathrm{mL}$ ) were prepared with PBS.

\section{Cell viability assay}

DU145 cells were seeded onto 24 -well plates at a density of $5 \times$ $10^{5}$ cells $/ \mathrm{mL}$, and then treated with the indicated concentrations of UDCA for 24 hours and 48 hours. MTT $(0.5 \mathrm{mg} / \mathrm{mL})$ was subsequently added to each well. After 3 hours of additional incubation, $100 \mu \mathrm{L}$ of a solution containing $10 \%$ SDS (pH 4.8) plus $0.01 \mathrm{~N} \mathrm{HCl}$ was added to dissolve the crystals. The absorbance at $570 \mathrm{~nm}$ were determined with an ELISA plate reader.

\section{Flow cytometric analysis}

After treatment of UDCA for 24 hours, the cells were collected, washed with cold PBS, and then centrifuged. The pellet was fixed in $75 \%(\mathrm{v} / \mathrm{v})$ ethanol at $4^{\circ} \mathrm{C}$ for 1 hour. The cells were washed once with PBS and re-suspended in cold PI solution $(50 \mu \mathrm{g} / \mathrm{mL})$ containing RNase A $(0.1 \mathrm{mg} / \mathrm{mL})$ in PBS (pH 7.4) for 30 minutes in the dark. The stained cells were analyzed by fluorescent activated cell sorting (FACS) for relative DNA content based on red fluorescence on a FACScan flow cytometer (Becton Dickinson, San Jose, CA, USA).

\section{Western blot analysis}

DU145 cells were treated with indicated concentrations of UDCA for indicated times at $37^{\circ} \mathrm{C}$ and cells were lysed in ice-cold radioimmunoprecipitation assay buffer ( $1 \%[\mathrm{w} / \mathrm{w}] \mathrm{NP} 40,1 \%[\mathrm{w} / \mathrm{v}]$ sodium deoxycholate, $0.1 \%[\mathrm{w} / \mathrm{v}$ ] SDS, $0.15 \mathrm{M} \mathrm{NaCl}, 0.01 \mathrm{M}$ sodium phosphate buffer, pH 7.2, 2 mM EDTA, $50 \mathrm{mM} \mathrm{NaF}$, and protease inhibitors). The cells were disrupted by sonication and extracted at $4^{\circ} \mathrm{C}$ for 30 minutes. The protein concentrations were quantified using the BioRad protein assay kit (BioRad Lab., Hercules, CA, USA). The proteins in the extracts were resolved by electrophoresis and electro-transferred to a polyvinylidene difluoride membrane (Millipore, Bedford, MA, USA). The membrane was incubated with the primary antibodies and a secondary antibodies conjugated to peroxidase. Blots were developed with an ECL detection system.

\section{Statistical analysis}

Results were expressed as means $\pm \mathrm{SD}$. Comparison of the effects of various treatments was performed by the one-way ANOVA with post-test Neuman-Keuls and Student t-test. Statistical significance was defined as $P<0.05$. Each experiment was performed at least in triplicate. A statistical analysis was performed using IBM SPSS ver. 21.0 software (IBM Co., Armonk, 
NY, USA).

\section{RESULTS}

\section{Ursodeoxycholic acid induces apoptosis in human DU145 prostate cancer cells}

To investigate anti-cancer activity of UDCA, DU145 cells were treated with various concentrations of UDCA (0 to $200 \mu \mathrm{g} / \mathrm{mL}$ ) for 24 hours and 48 hours. Cell growth was assessed by an MTT assay. The MTT assay revealed that the growth of DU145 cells was inhibited by UDCA treatment in a dose-dependent manner, and the $50 \%$ inhibition of cell growth $\left(\mathrm{IC}_{50}\right)$ was approximately 200 $\mu \mathrm{g} / \mathrm{mL}$ (Fig. 1A). To determine whether the decreased cell viability was due to apoptotic cell death, we assessed the changes in cellular morphology of UDCA-treated cells under a light microscope. Cellular shrinkage and cytoplasmic blebs were observed at the concentration of $100 \mu \mathrm{g} / \mathrm{mL}$ or higher (Fig. 1B). Next, we analyzed the magnitude of apoptotic cell death and cell cycle distribution in UDCA-treated cells by flow cytometry. As shown in Figure 2, UDCA induced in a significant accumulation of cells with sub-G1 DNA content at the concentration of $200 \mu \mathrm{g} / \mathrm{mL}$. These results suggest that the cytotoxic effects observed in response to UDCA are associated with the induction of apoptotic cell death in human DU145 prostate cancer cells.

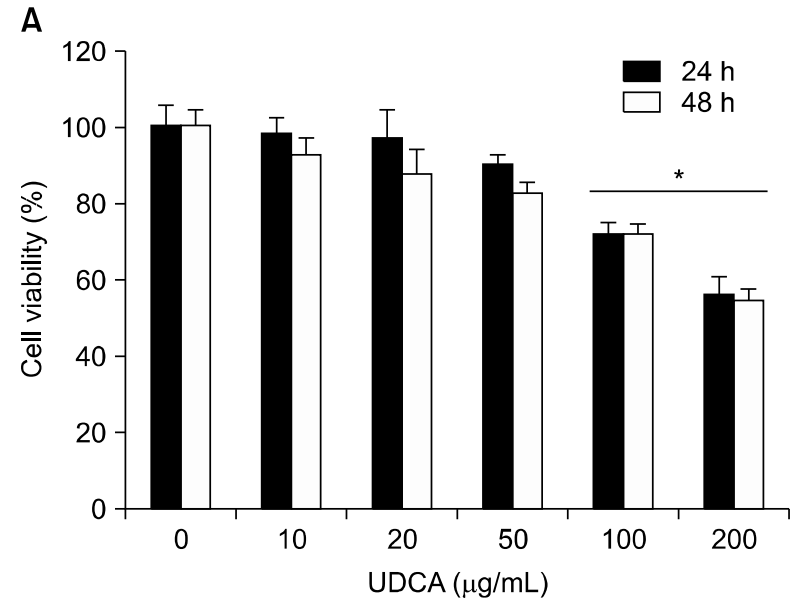

B

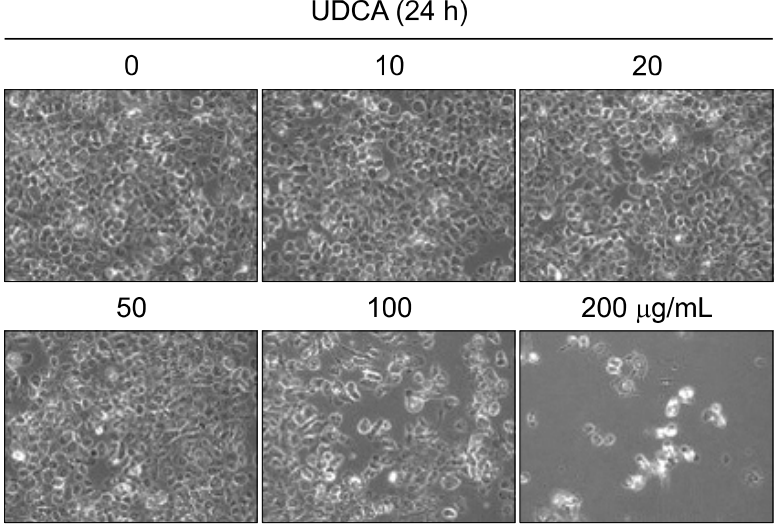

Figure 1. Ursodeoxycholic acid (UDCA) reduces the viability of DU145 cells. The cells were seeded at the density of $1 \times 10^{5}$ cells $/ \mathrm{mL}$ and incubated with the indicated concentrations of UDCA for 24 hours and 48 hours. (A) Cell viability was assessed by an MTT assay. (B) The morphology of UDCA-treated DU145 cells was observed under a light microscope (magnification $\times 200$ ). Data are expressed as mean \pm SD of three independent experiments $(* P<0.05$ versus control).

A

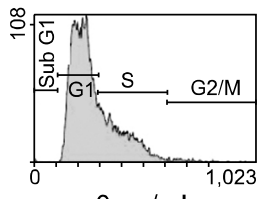

$0 \mu \mathrm{g} / \mathrm{mL}$

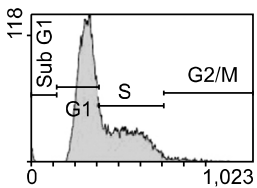

$50 \mu \mathrm{g} / \mathrm{mL}$

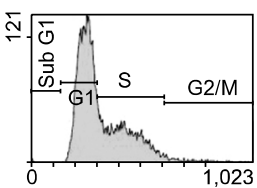

$10 \mu \mathrm{g} / \mathrm{mL}$

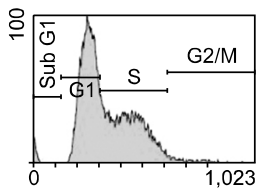

$100 \mu \mathrm{g} / \mathrm{mL}$

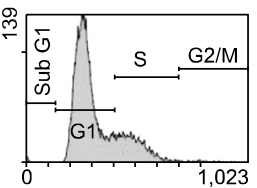

$20 \mu \mathrm{g} / \mathrm{mL}$

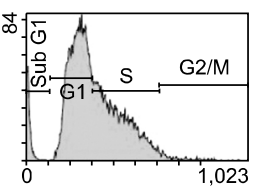

$200 \mu \mathrm{g} / \mathrm{mL}$
B

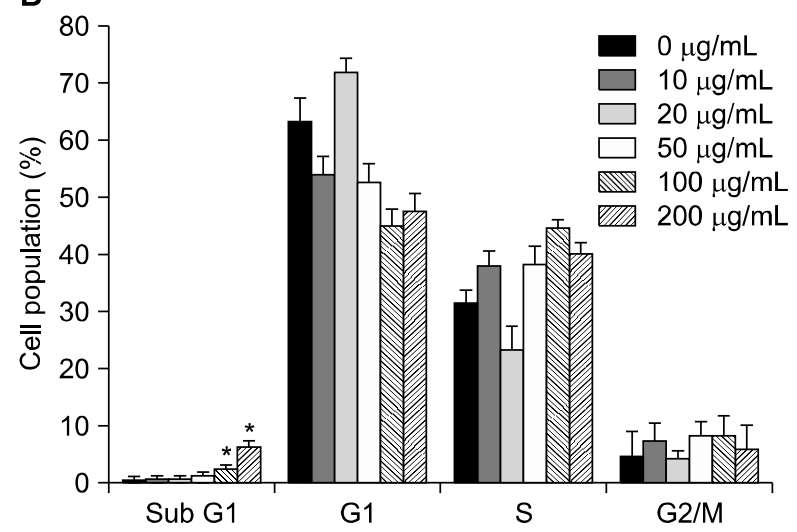

Figure 2. Ursodeoxycholic acid (UDCA) induces apoptotic cell death in DU145 cells. The cells were incubated with the indicated concentrations of UDCA for 24 hours and stained with propidium iodide. (A) The cells with sub-G1 DNA content representing apoptotic DNA degradation were analyzed by flow cytometry. (B) The results are from at least three independent experiments that showed similar patterns $(* P<0.05$ versus control). 
2. Ursodeoxycholic acid induces caspase-dependent apoptosis and subsequent cleavage of PARP in DU145 cells

We then assessed the effect of UDCA on caspases and their substrates, PARP by Western blot analysis. UDCA activated caspase- 3 and caspase- 8 in a dose- and time-dependent manner. With the activation of caspases, the cleavage of PARP was increased (Fig. 3). Further, the expression of pro-apoptotic protein Bax was significantly increased; meanwhile anti-apoptotic protein Bcl-xL was significantly decreased in a dose- and time-dependent manner. These pro-apoptotic and anti-apoptotic proteins involves in the intrinsic apoptotic pathway, which creates pores in the mitochondrial outer membrane, and releases cytochrome $c$ into the cytoplasm, and activates caspases that results in cell death. ${ }^{8}$ UDCA significantly increased cytochrome $c$ in DU145 cells in a dose- and time-dependent manner. These findings suggest that UDCA may induce apoptosis through intrinsic apoptotic pathway.

\section{Ursodeoxycholic acid induces death receptor-me- diated apoptosis in DU145 cells}

To further investigate the mechanisms of the UDCA-induced apoptosis, we investigated the expression of TRAIL receptors. Western blot analysis revealed that the expression of DR4 and DR5 was significantly up-regulated by UDCA (Fig. 3). This finding suggests that UDCA-induced apoptosis may be associated the death receptor-mediated apoptosis in DU145 cells.
4. Tumor necrosis factor-related apoptosis-inducing ligand augments the ursodeoxycholic acid-induced cell death in DU145 cells

Next, we determined whether TRAIL can enhance the UDCA-induced anti-cancer effect. MTT assay and morphology test revealed that UDCA could augment the TRAIL-induced cell death (Fig. 4A and 4B). Western blot results confirmed that TRAIL treatment up-regulated DR4 and DR5 expressions (Fig. 4C). These findings suggest that TRAIL may promote UDCA-induced apoptosis in DU145 cells.

\section{DISCUSSION}

This study was designed to determine whether UDCA has anti-cancer properties in human prostate cancer cells, and to further investigate the underlying mechanisms of the anti-cancer effect of UDCA. We demonstrated that UDCA induced apoptosis in a dose-dependent manner in human DU145 cells.

Firstly, UDCA significantly inhibited the growth, and shrinking cells and cytoplasmic blebs were observed in DU145 cells. These results are consistent with previous report that biochemical and morphological changes occur during the apoptosis process. ${ }^{9}$ To determine how UDCA induces apoptosis, Western blot analysis was performed. We found that UDCA induced pro-apoptotic Bax protein whereas it reduced the expression of the anti-apoptotic Bcl-xL protein. UDCA also increased the expression of cytochrome $c$ in UDCA-treated DU145 cells. The evidence suggests
A

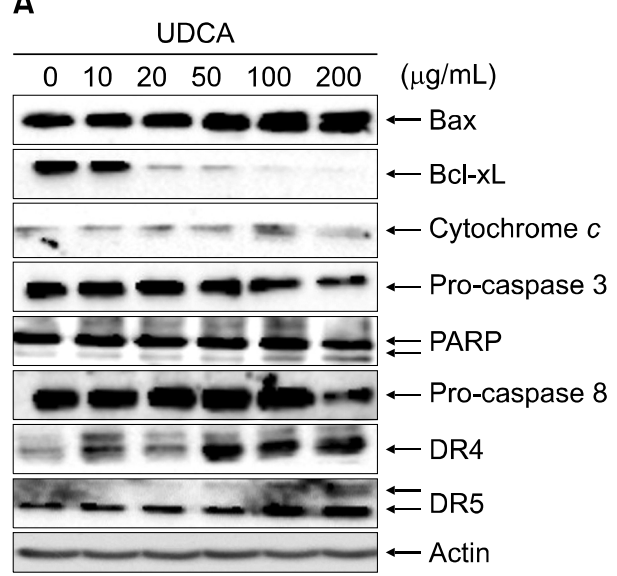

B

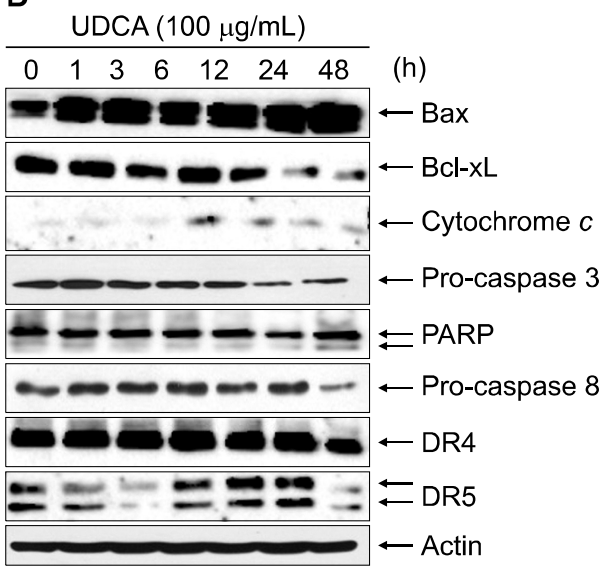

Figure 3. Ursodeoxycholic acid (UDCA)-induces caspase-dependent apoptosis and subsequent cleavage of PARP in human DU145 human prostate cancer cells. (A) The cells were treated with UDCA for 24 hours at the indicated concentrations, and (B) for indicated time intervals at $100 \mu \mathrm{g} / \mathrm{mL}$ of UDCA. Equal amounts of cell lysate $(30 \mu \mathrm{g})$ were resolved by SDS-PAGE gels and transferred onto nitrocellulose membranes. The membranes were probed with the indicated antibodies and the expression of target proteins was detected by the enhanced chemiluminescence system. The results are from at least three independent experiments that showed similar patterns. DR4, death receptor 4; DR5, death receptor 5 . 
A

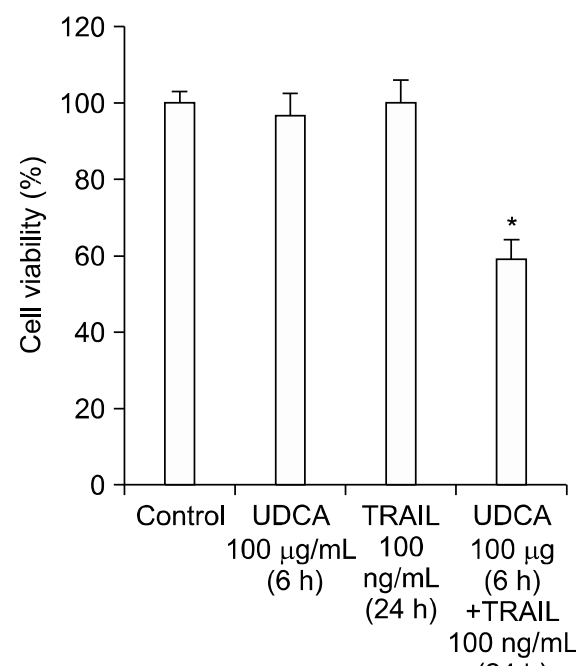

B

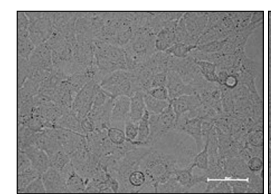

Control

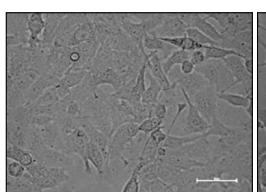

UDCA

$100 \mu \mathrm{g} / \mathrm{mL}(6 \mathrm{~h})$

$(24 \mathrm{~h})$

C

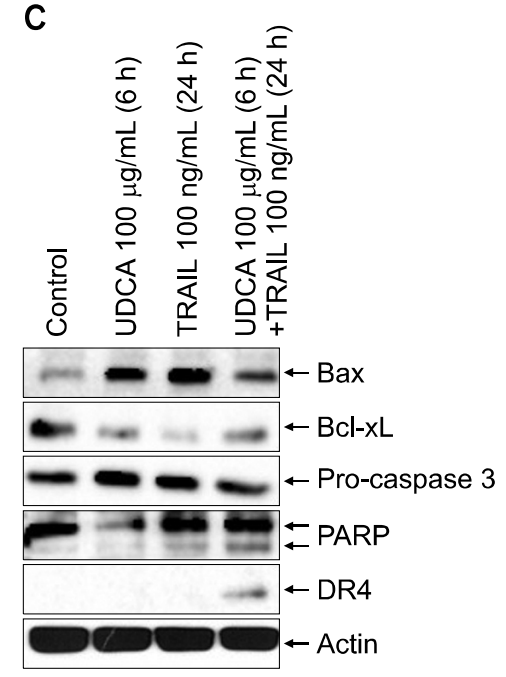

Figure 4. Tumor necrosis factor-related apoptosis-inducing ligand (TRAIL) augments the ursodeoxycholic acid (UDCA) induced cell death in DU145 cells. The cells were incubated at indicated concentrations of UDCA and TRAIL for indicated time intervals. (A) Cell viability was assessed by MTT assay. (B) The morphology of the DU145 cells was observed using light microscope (magnification, $\times 400$ ). (C) Equal amounts of cell lysate $(30 \mu \mathrm{g})$ were resolved by SDS-PAGE gels and transferred onto nitrocellulose membranes. The membranes were probed with the indicated antibodies and target proteins were detected by the enhanced chemiluminescence system. Data are expressed as mean \pm SD of three independent experiments $(* P<0.05$ versus control). DR4, death receptor 4.

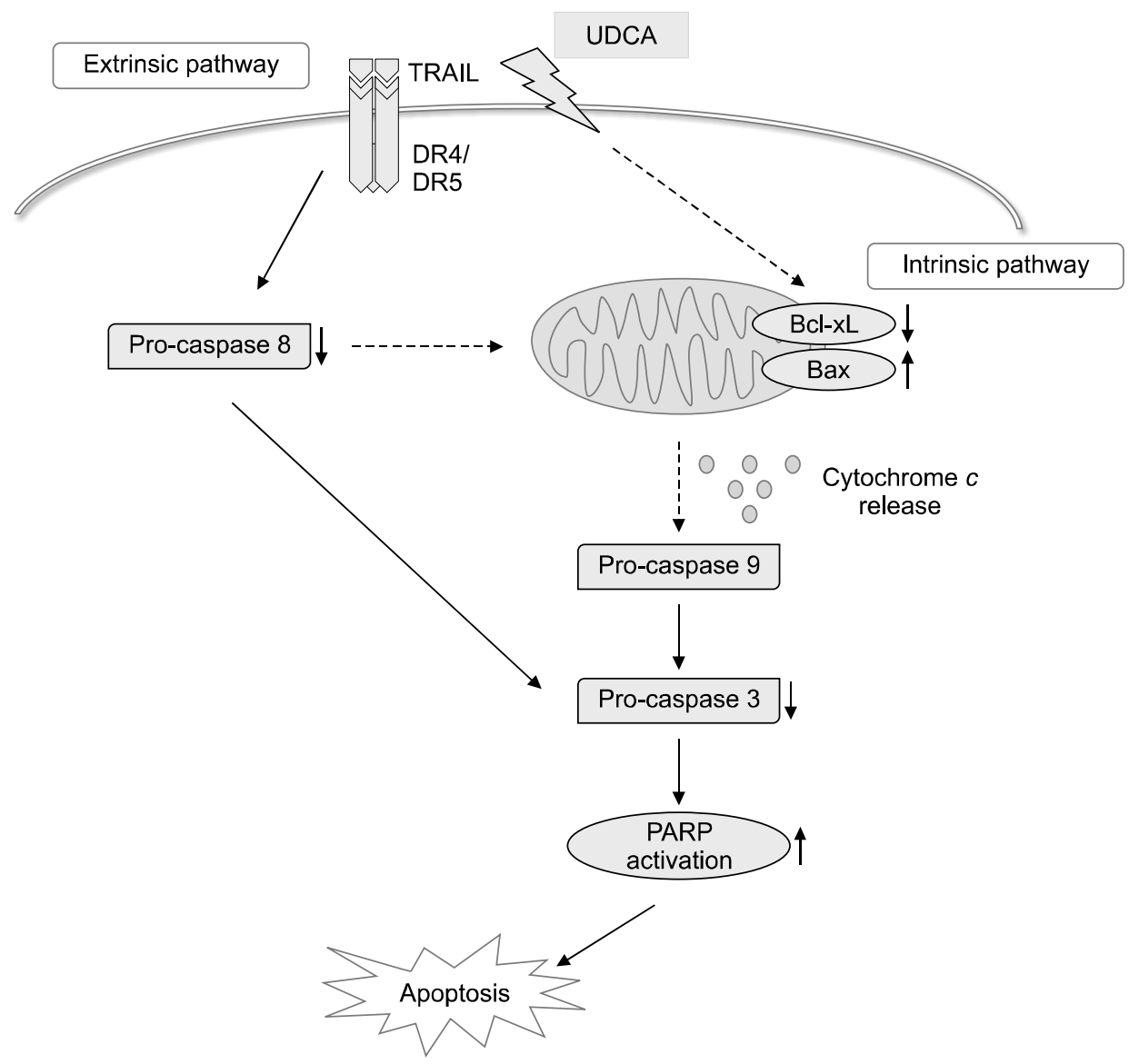

Figure 5. Schematic representation of ursodeoxycholic acid (UDCA)-induced apoptosis in DU145 human prostate cancer cells. The apoptosis was triggered through extrinsic pathway by up-regulating tumor necrosis factor-related apoptosis-inducing ligand (TRAIL) receptor, death receptor 4 (DR4) and death receptor 5 (DR5). As well as through intrinsic pathway by modulating Bax and Bcl-xL levels and cytochrome $c$ release $\rightarrow$ indicates activation, $\rightarrow$ indicates indirect or multiple pathways, $\uparrow$ indicates up-regulation, and $\downarrow$ indicates down-regulation). 
that the changes in the expression of pro-apoptotic and anti-apoptotic proteins are involved in the intrinsic apoptotic pathway. Moreover, UDCA increased the expression of DR4 and DR 5 and UDCA-induced cell death was promoted by TRAIL treatment in DU145 cells. These findings support that UDCA induces apoptosis via both the intrinsic and extrinsic pathways. In addition, the increased DR5 expression has been highly correlated with sensitivity to TRAIL, ${ }^{10}$ and over-expression of DR5 in TRAIL-resistant cancer cells restored TRAIL sensitivity. ${ }^{11}$ Previously, we also reported that up-regulating DR5 is an important mechanism for apoptosis induced by natural compounds. $^{12,13}$

The limitation of this study is that the only one cell line was used in this study. Therefore, it may be hard to generalize these results. In addition, it is reported that UDCA effects may differ depending on cell types, physiologic conditions, and/or stimulus. $^{7,14,15}$ However, we also found that UDCA have anti-cancer effect on PC-3 cells even though the up-regulation of TRAIL receptor DR4 and DR5 was not clearly detected (data not shown).

In summary, UDCA induces apoptosis in human DU145 prostate cancer cells (Fig. 5). The apoptosis is triggered by extrinsic pathway through up-regulation of TRAIL receptor DR4 and DR5, as well as intrinsic pathway through modulation of Bax, Bcl-xL and cytochrome $c$ release. This study provides evidence that UDCA might have anti-cancer property on human prostate cancer cells, and it can be used as an alternative medical agent for the treatment of prostate cancer.

\section{ACKNOWLEDGMENTS}

This work was supported by the grants from the National R \& D Program for Cancer Control, Ministry of Health \& Welfare, Republic of Korea (0820050).

\section{CONFLICTS OF INTEREST}

No potential conflicts of interest were disclosed.

\section{REFERENCES}

1. Mahon KL, Henshall SM, Sutherland RL, Horvath LG. Pathways of chemotherapy resistance in castration-resistant prostate cancer. Endocr Relat Cancer 2011;18:R103-23.

2. Lefebvre P, Cariou B, Lien F, Kuipers F, Staels B. Role of bile acids and bile acid receptors in metabolic regulation. Physiol Rev 2009;89:147-91.

3. Thomas C, Auwerx J, Schoonjans K. Bile acids and the membrane bile acid receptor TGR5--connecting nutrition and metabolism. Thyroid 2008; 18:167-74.

4. Goldberg AA, Titorenko VI, Beach A, Sanderson JT. Bile acids induce apoptosis selectively in androgen-dependent and -independent prostate cancer cells. PeerJ 2013;1:e122.

5. Lim SC, Duong HQ, Choi JE, Lee TB, Kang JH, Oh SH, et al. Lipid raft-dependent death receptor 5 (DR5) expression and activation are critical for ursodeoxycholic acid-induced apoptosis in gastric cancer cells. Carcinogenesis 2011;32:723-31.

6. Lim SC, Choi JE, Kang HS, Han SI. Ursodeoxycholic acid switches oxaliplatin-induced necrosis to apoptosis by inhibiting reactive oxygen species production and activating p53-caspase 8 pathway in HepG2 hepatocellular carcinoma. Int J Cancer 2010;126:1582-95.

7. Kessel D, Caruso JA, Reiners JJ Jr. Potentiation of photodynamic therapy by ursodeoxycholic acid. Cancer Res 2000;60:6985-8.

8. Li P, Nijhawan D, Budihardjo I, Srinivasula SM, Ahmad M, Alnemri ES, et al. Cytochrome $\mathrm{c}$ and dATP-dependent formation of Apaf-1/caspase-9 complex initiates an apoptotic protease cascade. Cell 1997:91:479-89.

9. Majno G, Joris I. Apoptosis, oncosis, and necrosis. An overview of cell death. Am J Pathol 1995;146:3-15.

10. Jang YJ, Park KS, Chung HY, Kim HI. Analysis of the phenotypes of Jurkat clones with different TRAIL-sensitivities. Cancer Lett 2003:194:107-17.

11. Mitsiades N, Poulaki V, Mitsiades C, Tsokos M. Ewing's sarcoma family tumors are sensitive to tumor necrosis factor-related apoptosis-inducing ligand and express death receptor 4 and death receptor 5. Cancer Res 2001;61:2704-12.

12. Han MH, Lee WS, Jung JH, Jeong JH, Park C, Kim HJ, et al. Polyphenols isolated from Allium cepa L. induces apoptosis by suppressing IAP-1 through inhibiting PI3K/Akt signaling pathways in human leukemic cells. Food Chem Toxicol 2013;62:382-9.

13. Jeong JW, Lee WS, Go SI, Nagappan A, Baek JY, Lee JD, et al. Pachymic acid induces apoptosis of EJ bladder cancer cells by DR5 up-regulation, ROS generation, modulation of Bcl-2 and IAP family members. Phytother Res 2015;29:1516-24.

14. Milovic V, Teller IC, Faust D, Caspary WF, Stein J. Effects of deoxycholate on human colon cancer cells: apoptosis or proliferation. Eur J Clin Invest 2002;32:29-34.

15. Im E, Martinez JD. Ursodeoxycholic acid (UDCA) can inhibit deoxycholic acid (DCA)-induced apoptosis via modulation of EGFR/Raf-1/ERK signaling in human colon cancer cells. J Nutr 2004:134:483-6. 